\title{
Cognitive decline following psychosis onset
}

\author{
Data from the PACE clinic
}

STEPHEN J. WOOD, WARRICK J. BREWER, PENNY KOUTSOURADIS,
LISA J. PHILLIPS, SHONA M. FR ANCEY, TINA M. PROFFITT, ALISON R. YUNG,
HENRY J. JACKSON, PATRICK D. MCGORRY and CHRISTOS PANTELIS

Background The origin of cognitive impairments in psychotic disorders is still unclear. Although some deficits are apparent prior to the onset of frank illness, it is unknown if they progress.

Aims To investigate whether cognitive function declined over the transition to psychosis in a group of ultra-high risk individuals.

Method Participants consisted of two groups: controls $(n=17)$ and individuals at ultra-high risk for development of psychosis $(n=16)$. Seven of the latter group later developed psychosis.

Neuropsychological testing was conducted at baseline and again after at least a 12-month interval.

Results Both the Visual Reproduction sub-test of the Wechsler Memory ScaleRevised and Trail-Making Test B showed a decline over the follow-up period that was specific to the group who became psychotic. In addition, both high-risk groups showed a decline in digit span performance. No other task showed significant change over time.

Conclusions These preliminary data suggest that as psychosis develops there may be a specific decline in visual memory and attentional set-shifting, reflecting impairments in efficient organisation of visual stimuli. This may be caused by either the illness itself or treatment with antipsychotic medication.

Declaration of interest None. Funding detailed in Acknowledgements.
Cognitive impairments, predominantly in attention, memory, and executive functions, are commonly identified in early psychosis (Bilder et al, 2000; Addington et al, 2003), and appear to remain stable over time (Hoff et al, 1999; Addington et al, 2005). Currently there is no clear understanding of the course of cognitive dysfunction prior to the onset of psychosis. Although individuals who later develop schizophrenia may exhibit cognitive abnormalities during childhood (Johnstone et al, 2002; Conklin \& Iacono, 2003; for review see Niemi et al, 2003), studies of people at ultra-high risk for psychosis (defined by a mix of trait and state factors) have generally found deficits considerably less profound than those seen in the first episode (Brewer et al, 2005; Lencz et al, 2006; for review, see Brewer et al, 2006). Further, recent evidence suggests there are active brain structural changes over the transition to psychosis in high-risk groups, involving temporal and frontal lobe regions (Pantelis et al, 2003; Job et al, 2005). Taken together, these findings suggest that cognitive performance may show a definite decline over the transition from the at-risk mental state to frank psychosis. In this study, we aimed to investigate whether cognitive function declined over the transition to psychosis in a small sample of participants with ultra-high risk. Given our previous findings and those of others, we predicted that there would be a significant decline in memory and executive function that was specific to the group who developed psychosis.

\section{METHOD}

\section{Participants}

Participants consisted of two groups comprising a total of 33 people, as follows: 17 control participants recruited by approaching ancillary hospital staff and their families, and by advertisements in local newspapers and bulletins, and 16 individuals at ultra-high risk for development of psychosis recruited from the Personal Assessment and Crisis Evaluation (PACE) Clinic (Yung et al, 2003, 2004). All but 8 of the participants were part of a larger, previously described sample (Brewer et al, 2005) (the 8 were all PACE clients seen after August 1998). There were no differences between those who were and those who were not followed up except that the follow-up group were non-significantly younger (mean age at baseline 18.8 years compared with 20.3 years, $P=0.07$ ).

The criteria for identification of the ultra-high risk group have been previously described (Yung et al, 2003, 2004); briefly, participants met the criteria for one or more of the following categories at intake: trait plus state risk factors; attenuated psychotic symptoms; or brief limited intermittent psychotic symptoms (BLIPS). The criteria met by the participants at ultra-high risk were as follows: trait plus state $(n=3)$, attenuated symptoms $(n=8)$, BLIPS $(n=2)$, trait plus state and attenuated symptoms $(n=1)$, attenuated symptoms and BLIPS $(n=1)$, and all three categories $(n=1)$. All participants at ultra-high risk were between the ages of 14 and 29 years at baseline, had not experienced a previous psychotic episode (treated or untreated), and reported English as the preferred language. In order to identify the onset of acute levels of psychosis in the ultra-high risk group, operationalised criteria for the onset of psychosis have been defined (Yung et al, 2003, 2004). Individuals at ultra-high risk were divided into two subgroups, depending on psychotic status at the follow-up assessment: ultra-high risk with psychosis $(n=7)$ and ultra-high risk with no psychosis $(n=9)$.

The psychotic diagnoses in the ultrahigh risk with psychosis group at followup - using the Diagnostic and Statistical Manual of Mental Disorders (DSM-IV; American Psychiatric Association, 1994) were schizophrenia and schizophreniform psychosis $(n=4)$, brief psychotic disorder $(n=1)$, bipolar disorder with psychotic features $(n=1)$, and psychotic disorder not otherwise specified $(n=1)$. The majority of the ultra-high risk with no psychosis group had no current axis I diagnosis at follow-up $(n=5)$, while the remaining 4 participants were diagnosed with dysthymia $(n=1)$, obsessive-compulsive disorder $(n=1)$, major depressive disorder $(n=1)$, and generalised anxiety disorder $(n=1)$. One of the 
participants from the ultra-high risk with no psychosis group received antipsychotic treatment ( $2 \mathrm{mg}$ risperidone) following the baseline assessment as part of an intervention study exploring the effects of risperidone and psychotherapy on rate of transition to psychosis (McGorry et al, 2002). No other participant from this group took antipsychotic medication. All individuals from the ultra-high risk with psychosis group were prescribed antipsychotics after transition to frank psychosis; however, data concerning the type and dose were unavailable for 3 patients. Of the remaining 4, 2 were no longer taking medication at the time of reassessment, 1 was receiving risperidone and 1 chlorpromazine.

Participants were excluded from the study if they had documented neurological disorder; past history of head injury with loss of consciousness; impaired thyroid function; steroid use or DSM-IV diagnosis of alcohol dependence; estimated premorbid IQ below 70 (i.e. intellectual disability); or for healthy control participants, a personal history of axis I psychiatric illness or documented family history of psychotic illness. Written informed consent from the participants was obtained in accordance with the local research and ethics committee guidelines.

\section{Measures}

\section{Premorbid IQ}

The National Adult Reading Test (NART; Nelson \& Willison, 1991) provided an estimate of premorbid intellectual ability. Australian norms adjusted for educational level (Willshire et al, 1991) were used to calculate subject scores.

\section{Current IQ}

Four subtests of the Wechsler Adult Intelligence Scale - Revised (WAIS-R, Wechsler, 1981) were administered in order to calculate the Kaufman 4-test short-form IQ score (i.e. arithmetic, similarities, picture completion and digit symbol) (Kaufman, 1990). In addition, the information, digit span and block design sub-tests were administered.

\section{Attention and executive functioning}

The Controlled Oral Word Association Test (COWAT, Benton \& Hamsher, 1983) provided a measure of verbal fluency, and the Trail-Making Test parts A and B (Adjutant General's Office, 1944) assessed visuomotor speed and taskswitching ability.

\section{Learning and memory}

Sub-tests (logical memory I, paired associates I and visual reproduction I) from the Wechsler Memory Scale - Revised (WMS-R; Wechsler, 1987) provided measures of new verbal learning and of visual and verbal memory function. The Rey Auditory Verbal Learning Test (RAVLT, Rey, 1941) modified to three trials was used to assess new verbal learning capacity and delayed recall.

Fully qualified neuropsychologists or clinical psychologists conducted all research assessments at baseline and at follow-up. Owing to time constraints or compliance problems, not all participants completed all tests at both time points.

\section{Statistical analysis}

All data were analysed using SPSS for Mac, version 11. Demographic variables were compared using $\chi^{2}$ (male/female ratio), one-way analysis of variance (ANOVA; age, NART IQ, time between assessments) or $t$-tests (Brief Psychiatric Rating Scale, Schedule for the Assessment of Negative Symptoms). A series of two-way repeated measures ANOVAs was conducted to compare change in neuropsychological test scores over time. Because group effects have already been reported in a much larger sample (Brewer et al, 2005), only time and time $\times$ group effects are reported here. Agescaled scores or age-appropriate percentile scores were used where available to obviate the need for age covarying. However, these were unavailable for certain tests, so repeated measures ANCOVA was used instead (see below for details).

\section{RESULTS}

\section{Demographic variables}

Demographic details are shown in Table 1. The ultra-high risk with psychosis group was significantly younger than the ultrahigh risk with no psychosis group at both assessments, and also had lower levels of global symptoms at baseline (as assessed by the Brief Psychiatric Rating Scale). There were no differences between the groups in estimated premorbid IQ or in time between the assessments.

\section{Global cognitive function}

Baseline and follow-up data for current IQ and the WAIS-R sub-tests are shown in Table 2. There was no significant effect of time and no significant time $\times$ group interaction for current IQ. Examination of the WAIS-R sub-tests revealed no significant main effect of time or time $\times$ group

Table I Demographic details for all groups

\begin{tabular}{|c|c|c|c|c|}
\hline & $\begin{array}{l}\text { Control } \\
\text { group }\end{array}$ & $\begin{array}{l}\text { Ultra-high risk with } \\
\text { psychosis group }\end{array}$ & $\begin{array}{l}\text { Ultra-high risk without } \\
\text { psychosis group }\end{array}$ & $P$ \\
\hline Gender, male/female & $14 / 3$ & $5 / 2$ & $5 / 4$ & 0.343 \\
\hline Age at baseline, years (s.d.) & $19.7(2.4)$ & $17.3(2.8)$ & $21.0(3.1)$ & 0.038; UHR-P $<$ UHR-NP \\
\hline Age at second assessment, years (s.d.) & $21.2(2.6)$ & $18.3(2.7)$ & $22.5(3.2)$ & $0.018 ;$ UHR-P $<$ UHR-NP \\
\hline NART-estimated IQ at baseline, mean score (s.d.) & $110.4(9.5)$ & $105.3(15.3)$ & $107.5(14.5)$ & 0.623 \\
\hline Time between assessments, days (s.d.) & $560(263)$ & $372(175)$ & $575(327)$ & 0.252 \\
\hline Days between baseline and onset of psychosis, mean (s.d.) & - & $257(174)$ & - & - \\
\hline Days between onset of psychosis and follow-up, mean (s.d.) & - & $116(95)$ & - & - \\
\hline BPRS at intake, mean score (s.d.) & - & I5.I (5.4) & $28.3(13.7)$ & 0.031 \\
\hline SANS at intake, mean score (s.d.) & - & $23.7(16.5)$ & $20.7(13.1)$ & 0.686 \\
\hline
\end{tabular}

NART, National Adult Reading Test; BPRS, Brief Psychiatric Rating Scale; SANS, Schedule for the Assessment of Negative Symptoms. 


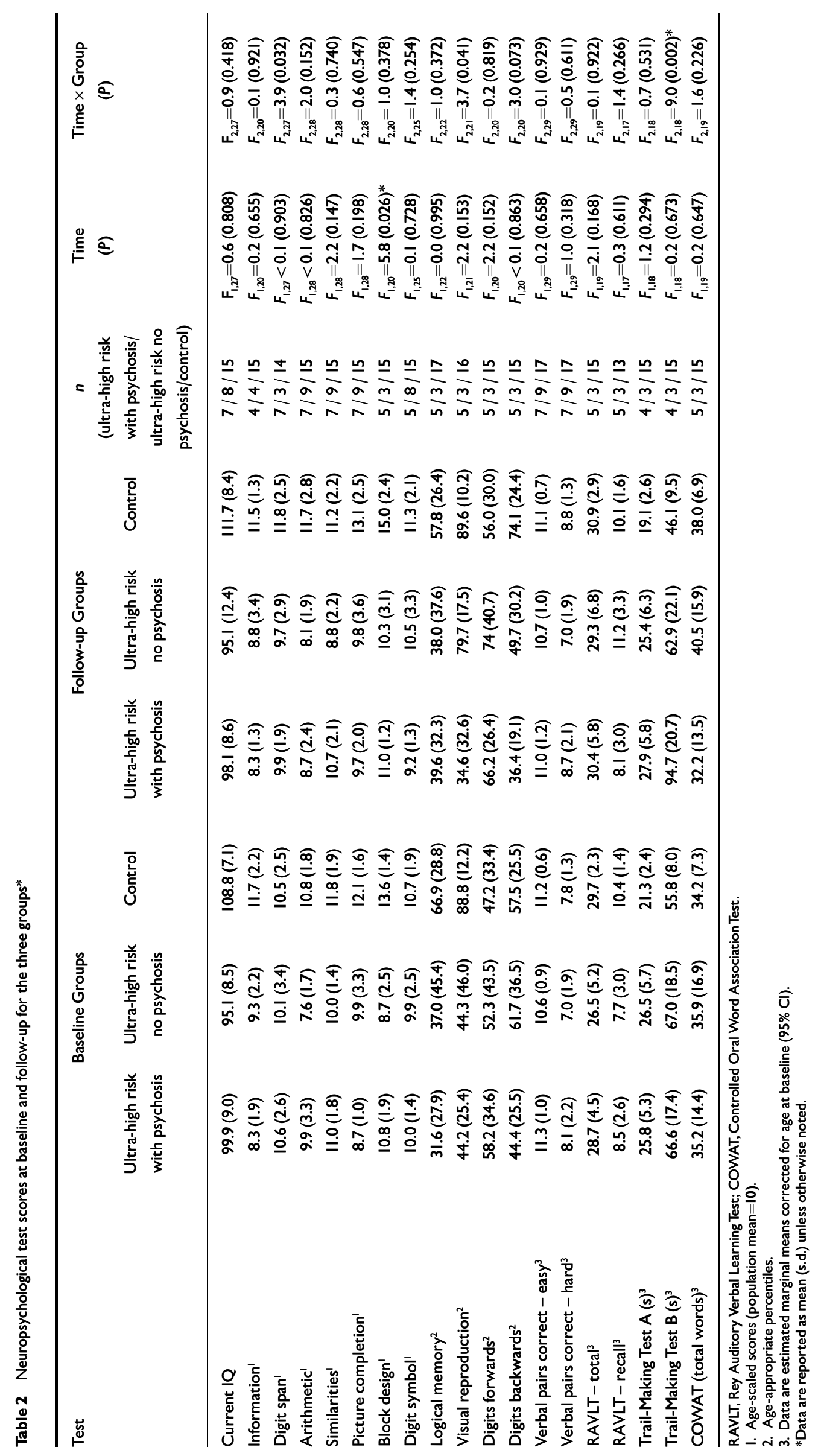


interaction for information, arithmetic, similarities, picture completion or digit symbol. A significant effect of time was found for block design (all groups improved at follow-up), and a significant time $\times$ group interaction was found for digit span. Posthoc tests revealed that only the control group showed a significant improvement over time $(P=0.045)$, while the two ultrahigh risk groups showed no significant change $(P=0.253$ and $P=0.347$ respectively).

\section{Memory}

Baseline and follow-up data for the WMS$\mathrm{R}$ sub-tests and the RAVLT are shown in Table 2. No main effects of time or time $x$ group interactions were found for the logical memory, digits forward or the digits backward sub-tests from the WMS-R. However, there was a significant time $x$ group interaction for the visual reproduction sub-test. Inspection of the data showed that although the ultra-high risk with no psychosis group improved markedly over the follow-up interval, the ultrahigh risk with psychosis group showed a decline in function (Fig. 1).

Because no age-corrected scores were available for the verbal paired associates easy, the verbal paired associates - hard or the RAVLT, repeated-measures ANCOVA (controlling for age at baseline) were performed. There were no significant main effects of time or time $\times$ group interactions for any of these scores.

\section{Executive function}

Baseline and follow-up data for the COWAT and parts A and B of the TrailMaking Test are shown in Table 2. For both tests, age was covaried as age-appropriate percentiles were unavailable. No main effect of time or time $\times$ group interaction was found for COWAT or for Trail-Making Test A. However, a significant time $\times$ group interaction effect was found for Trail-Making Test B, which was due to a large decline in the performance of the ultra-high risk with psychosis group. Both the ultra-high risk with no psychosis and the Control group showed slight improvements over the follow-up (Fig. 2).

\section{DISCUSSION}

To our knowledge, this is the first study to report longitudinal cognitive decline over the transition to psychosis in a clinical high-risk group. Although our data are preliminary, they suggest a specific decline in visual memory and attentional set-shifting, which may reflect impairments in efficient organisation of visual stimuli. Also, although both of the tasks that show specific decline in the ultra-high risk with psychosis group also involve motor and visuoconstructional skills, it is unlikely that these abilities are affected by the onset of psychosis, since neither Trail-Making Test A nor block design show the same change. One possibility is that the previously identified reduction in anterior cingulate grey matter over the transition to psychosis (Pantelis et al, 2003) is responsible for these cognitive changes. However, the onset of antipsychotic treatment in the ultra-high risk with psychosis group may also play a role in this decline.

These findings contrast with longitudinal studies of cognition in first-episode schizophrenia, which have consistently found no decline in performance over the years following illness onset (Hoff et al, 1999, 2005; Addington et al, 2005). Furthermore, a longitudinal study of cognition in a genetically at-risk group (the Edinburgh High Risk Study, Whyte et al, 2006) also found no specific decline over time in those who developed schizophrenia. Some evidence from cohort studies suggests that general cognitive decline occurs very early in the trajectory of illness, perhaps between late childhood and adolescence (Reichenberg et al, 2005). These differences with the current study are likely to be due to the ultra-high risk nature of the sample, and the fact that we were interested in transition to psychosis rather than schizophrenia generally.

Previously we have shown that the volume of the left medial temporal region reduces over the transition to psychosis (Pantelis et al, 2003). Given the role of this region in verbal associative memory (Eichenbaum, 1999), and the lack of a deficit prior to psychosis onset (Brewer et al, 2006), a decline in this cognitive function would be expected. Surprisingly, no
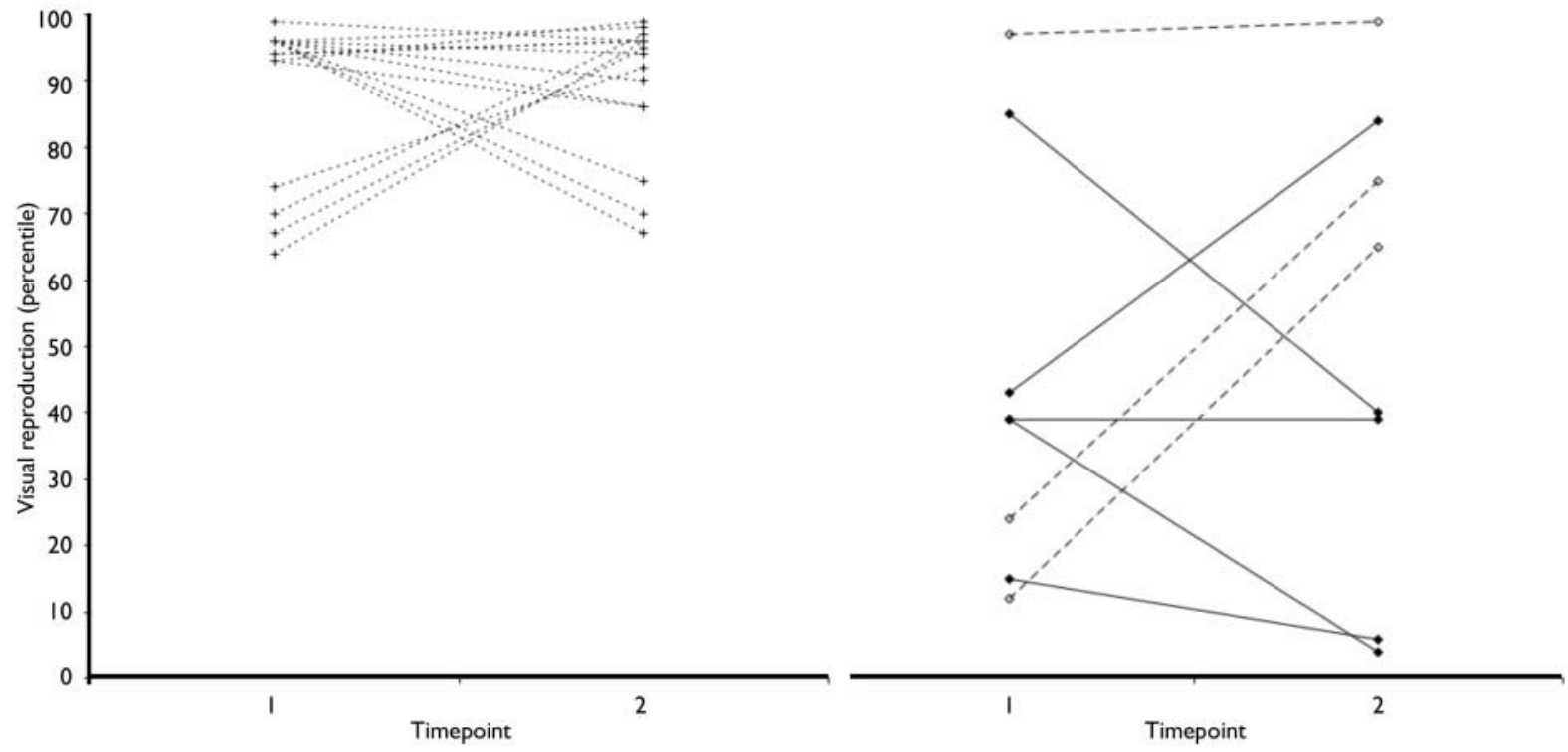

Fig. I Change in visual reproduction percentile for the three groups (left, control; right, UHR-P and UHR-NP). $-\downarrow$, ultra-high risk with psychosis group, - - $\diamond--$ ultrahigh risk with no psychosis group; - - + - - control group. 


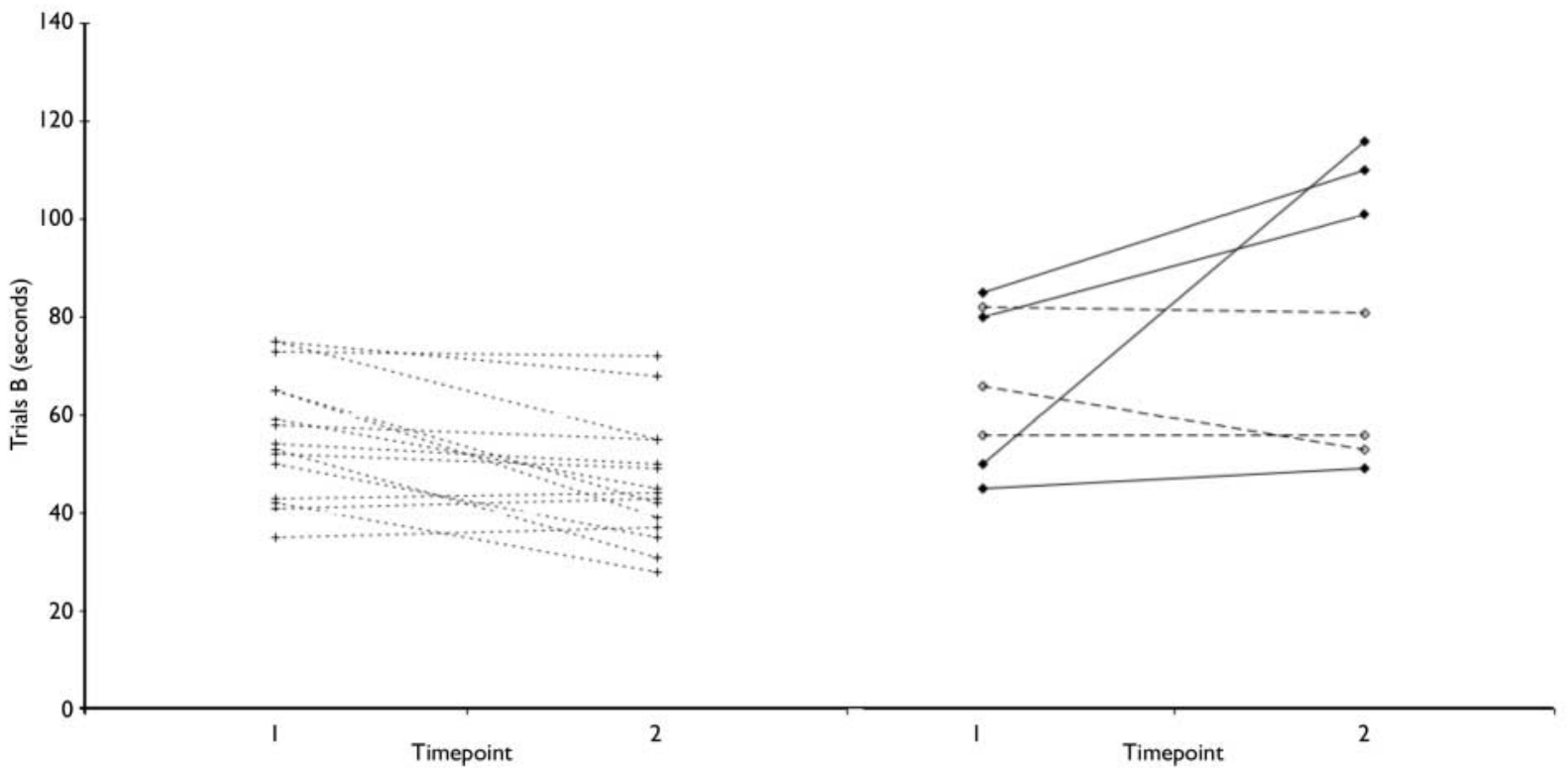

Fig. 2 Change in Trails B for the three groups (left, controls; right, UHR-P and UHR-NP). $-\longrightarrow$, ultra-high risk with psychosis group, - - -- ultra-high risk with no psychosis group; - - + - - control group.

such decline was identified. One possibility is that the tests we used here are not sensitive to pathology in this brain region. Alternatively, the volumetric change identified may be too small to cause detectable differences in memory performance.

The current study is clearly limited by the small sample size; for some sub-tests there were only data for 8 participants at ultra-high risk at both time points. Furthermore, although we did not find differences between these individuals and the larger sample previously reported, it is not clear that this group is necessarily representative even of the PACE group as a whole, let alone a more general group of people who develop first-episode psychosis. Therefore we cannot be certain that the cognitive declines seen in this study would be found in all people developing psychosis. Finally, we could not control treatment, either before or after transition to psychosis. It is therefore possible that antipsychotic medication may explain the decline in the ultra-high risk with psychosis group, although most findings in early psychosis are of improvement (for example, Keefe $e t$ $a l, 2006)$. However, a very recent report has suggested that 6 weeks of treatment with risperidone can impair spatial working memory ability in early psychosis (Reilly $e t$ al, 2006).

In summary, we have demonstrated specific cognitive decline over the transition to psychosis in tasks involving efficient organisation of visual stimuli, as a result either of the illness or of its treatment. Such a decline suggests a role for visual attentional systems in the onset of the disorder.

\section{ACKNOWLEDGMENTS}

This work was presented at the Australasian Society for Psychiatric Research meeting in Brisbane, December 2005. The work was supported by the NHMRC (Project Grant IDs: 970598 and 97039I, and Program Grant ID: 35024I). The PACE clinic was supported by grants from Janssen-Cilag, the Victor Hurley Medical Research Fund and a Research Program Grant from the Victorian Health Promotion Foundation. S.JW. is supported by an NHMRC Clinical Career Development Award and a NARSAD Young Investigator Award.

\section{REFERENCES}

Addington, J., Brooks, D. J. \& Addington, D. (2003) Cognitive functioning in first episode psychosis: initial presentation. Schizophrenia Research, 62, 59-64.

Addington, J., Saeedi, H. \& Addington, D. (2005)

The course of cognitive functioning in first episode psychosis: Changes over time and impact on outcome. Schizophrenia Research, 78, 35-43.

Adjutant General's Office (1944) Army Individual Test. Manual of Directions and Scoring. Washington DC: War Department.

American Psychiatric Association (1994) Diagnositic and Statistical Manual of Mental Disorders (4th edn). APA.

Benton, A. \& Hamsher, K. (1983) Multilingual Aphasia Examination. AJA Associates.

Bilder, R. M., Goldman, R. S., Robinson, D., et al (2000) Neuropsychology of first-episode schizophrenia: Initial characterization and clinical correlates. American Journal of Psychiatry, I57, 549-559.
Brewer, W. J., Francey, S. M., Wood, S. J., et al (2005) Memory impairments identified in people at ultra highrisk for psychosis who later develop first episode psychosis. American Journal of Psychiatry, 162, 71-78.

Brewer, W. J., Wood, S. J., Phillips, L. J., et al (2006) Generalized and specific cognitive performance in clinical high-risk cohorts: A review highlighting potentia vulnerability markers for psychosis. Schizophrenia Bulletin, 32, 538-555.

Conklin, H. M. \& lacono, W. G. (2003) At issue: assessment of schizophrenia: getting closer to the cause. Schizophrenia Bulletin, 29, 405-4II.

Eichenbaum, H. (1999) The hippocampus and mechanisms of declarative memory. Behavioural Brain Research, 103, 123-133.

Hoff, A. L., Sakuma, M., Wieneke, M., et al (1999) Longitudinal neuropsychological follow-up study of patients with first-episode schizophrenia. American Journal of Psychiatry, 156, 1336-1341.

Hoff, A. L., Svetina, C., Shields, G., et al (2005) Ten year longitudinal study of neuropsychological functioning subsequent to a first episode of schizophrenia. Schizophrenia Research, 78, 27-34.

Job, D. E., Whalley, H., Johnstone, E. C., et al (2005) Grey matter changes over time in high risk subjects developing schizophrenia. Neurolmage, 25, 1023-1030.

Johnstone, E. C., Cosway, R. \& Lawrie, S. M. (2002) Distinguishing characteristics of subjects with good and poor early outcome in the Edinburgh High-Risk Study. British Journal of Psychiatry, 43, S26-S29.

Kaufman, A. S. (1990) Assessing Adolescent and Adult Intelligence. Allyn \& Bacon, Inc.

Keefe, R. S., Young, C. A., Rock, S. L., et al (2006) One-year double-blind study of the neurocognitive efficacy of olanzapine, risperidone, and haloperidol in schizophrenia. Schizophrenia Research, 8I, I-I5.

Lencz, T., Smith, C. W., McLaughlin, D. B., et al (2006) Generalized and specific neurocognitive deficits in prodromal schizophrenia. Biological Psychiatry, 59, 863-87I. 
McGorry, P. D., Yung, A. R., Phillips, L. J., et al (2002) Randomized controlled trial of interventions designed to reduce the risk of progression to first-episode psychosis in a clinical sample with subthreshold symptoms. Archives of General Psychiatry, 59, 921-928.

\section{Nelson, H. E. \& Willison, J. R. (199I)}

Restandardisation of the NART against the WAIS-R. In National Adult Reading Test (NART) Test Manual (ed. H. E. Nelson), pp. 13-23. nFER-Nelson.

Niemi, L. T., Suvisaari, J. M., Tuulio-Henriksson, A., et al (2003) Childhood developmental abnormalities in schizophrenia: evidence from high-risk studies. Schizophrenia Research, 60, 239-258.

Pantelis, C., Velakoulis, D., McGorry, P. D., et al (2003) Neuroanatomical abnormalities before and after onset of psychosis: a cross-sectional and longitudinal MRI study. Lancet, 36I, 28I-288.

Reichenberg, A., Weiser, M., Rapp, M. A., et al (2005) Elaboration on premorbid intellectual performance in schizophrenia: Premorbid intellectual decline and risk for schizophrenia. Archives of General Psychiatry, 62, 1297-1304.

Reilly, J. L., Harris, M. S., Keshavan, M. S., et al (2006) Adverse effects of risperidone on spatial working memory in first-episode schizophrenia. Archives of General Psychiatry, 63, I189-1197.

Rey, A. (194I) Psychological examination of traumatic encephalopathy. Archives de Psychologie, 28, 286-340.

Wechsler, D. (198I) Wechsler Adult Intelligence Scale Revised Manual. Psychological Corporation.

STEPHEN J.WOOD, PhD, Melbourne Neuropsychiatry Centre, Department of Psychiatry, University of Melbourne, Australia,WARRICK J. BREWER, PhD, ORYGEN Research Centre, Department of Psychiatry, University of Melbourne, Australia, PENNY KOUTSOURADIS, BA, Post Grad Dip Psych, Melbourne Neuropsychiatry Centre, Department of Psychiatry, University of Melbourne and Department of Psychology, Australian Catholic University, Australia, LISA J. PHILLIPS, PhD, Department of Psychology, University of Melbourne, Australia, SHONA M. FRANCEY, PhD, TINA M. PROFFITT, DPsych, ALISON R. YUNG, MD, FRANZCP, Research Centre, Department of Psychiatry, University of Melbourne, Australia, HENRY J. JACKSON, PhD, Department of Psychology, University of Melbourne, Australia, PATRICK D. McGORRY, PhD, FRANZCP, ORYGEN Research Centre, Department of Psychiatry, University of Melbourne, Australia, CHRISTOS PANTELIS, MD, MRCPsych, FRANZCP, Melbourne Neuropsychiatry Centre, Department of Psychiatry, University of Melbourne, Australia

Correspondence: Dr Stephen Wood, Melbourne Neuropsychiatry Centre, c/o National Neuroscience Facility, I6I Barry Street, Carlton South, VIC 3053 Australia. tel: +6I 38344 1877; fax: +6I 393480469 ; email: sjwood@unimelb.edu.au

Wechsler, D. (1987) Wechsler Memory Scale - Revised. Psychological Corporation.

Whyte, M.-C., Brett, C., Harrison, L. K., et al (2006) Neuropsychological performance over time in people at high risk of developing schizophrenia and controls. Biological Psychiatry, 59, 730-739.

Willshire, D., Kinsella, G. \& Prior, M. (1991) Estimating WAIS-R IQ from the National Adult Reading
Test: a cross-validation. Journal of Clinical and Experimental Neuropsychology, 13, 204-216.

Yung, A. R., Phillips, L. J., Yuen, H. P., et al (2003) Psychosis prediction: 12 month follow-up of a high risk ('prodromal') group. Schizophrenia Research, 60, 21-32.

Yung, A. R., Phillips, L. J., Yuen, H. P., et al (2004) Risk factors for psychosis in an ultra high-risk group: psychopathology and clinical features. Schizophrenia Research, 67, $13 \mid-142$. 